Research Article

\title{
A Novel Balancing Method for Rotor Using Unsupervised Deep Learning
}

\author{
Shun Zhong $\mathbb{D}^{1}{ }^{1}$ Liqing Li $\left(\mathbb{D},{ }^{1}\right.$ Huizheng Chen, ${ }^{2}$ and Zhenyong $\mathrm{Lu}^{2}$ \\ ${ }^{1}$ Department of Mechanics and Key Laboratory of Dynamics and Control, Tianjin University, Tianjin, China \\ ${ }^{2}$ Institute of Dynamics and Control Science, Shandong Normal University, Jinan, China \\ Correspondence should be addressed to Liqing Li; li_qing_li@tju.edu.cn
}

Received 21 May 2021; Accepted 28 June 2021; Published 5 July 2021

Academic Editor: Yong-Feng Yang

Copyright (c) 2021 Shun Zhong et al. This is an open access article distributed under the Creative Commons Attribution License, which permits unrestricted use, distribution, and reproduction in any medium, provided the original work is properly cited.

A novel balancing method for rotor based on unsupervised deep learning is proposed in this paper. The architecture of the proposed deep network is described. In the proposed network, compared to the supervised deep network, additional convolution layers are applied not only for the learning of the inverse mapping but also for identifying the unbalanced force without labeled data. The equivalent value and position of imbalances in two correction planes are obtained. A case study of a rotor with two discs supported by sliding bearings is conducted. Preset imbalances are balanced well by the proposed method. And, using the state values at different time intervals, no extra weight trails are needed. The results show that the proposed balancing method gives consideration to both cost and accuracy.

\section{Introduction}

Rotor balancing is a type of analysis that compares the vibration profile with the rotation of a mechanical element to characterize inconsistent weight distribution around the diameter while calculating the amount and position of the weight necessary to offset the net imbalance. As rotor imbalance may lead to malfunction, such as rotor rub-impact and bearing wear, and even to catastrophic failure [1], the rotor balancing is a traditional technology, but still important in nowadays rotor industry. Any mass that is not rotating around its center of mass will produce vibration. Asymmetry of the structure along the rotating axis and small changes in density and thickness of the material cause imbalances. And, imbalance distribution leads to additional force and moment onto the rotor. Every single rotor needs several times of balancing like factory balancing [2] and onsite balancing [3] before implementation and online balancing [4] in working condition.

For decades, dynamic balancing methods are developed on the basis of two mature ideas. One is the modal balancing method (MBM) and the other is the influence coefficients method (ICM). Bishop [5] proposed the MBM at first, and it was optimized afterwards [6]. The ICM was proposed by Goodman [7] firstly and perfected by Lund and Tonnesen [8]. The central theory of the MBM is to balance the first $N$ modal unbalanced responses by adding trail weights to $N$ balance surfaces, and the speed of the system should be close to the critical speed of each mode. While for the ICM, the main technique is to identify the unbalanced parameters by the linear relationship between the trail weights and vibration responses of the rotor system.

Cost and accuracy are two main considering points in rotor balancing. Therefore, some dynamic balancing methods based on advanced technologies are proposed by researchers, and good performances with some specific cases were observed. ICM-like method [9-13] is easy to operate. However, more measuring data of the responses at different measuring points with different rotating speeds are usually necessary to achieve better performance. As for the ICM or like approaches, inappropriate position of measurement points may result in ill-conditioned coefficient matrix and lead to the failure of this method. Meanwhile, due to the limitation of the working conditions of the rotor system, high speed measures for long time may lead to decreasing of the system sensitivity at high modes. Thus, several times of 
weight trial processes are essential. MBM-like method [14-18] uses prior knowledge of the system to identify imbalance. But the change of the stiffness and damping, i.e. the support properties of bearings and high damping systems would affect identification results of rotor imbalances when balancing by the MBM. If we have enough prior knowledge about the rotor, the weight trail times can be reduced significantly by introducing the full set of modals.

Deep learning methods have attracted great attention in recent years and been applied in many fields, such as fault diagnosis of reciprocating compressor [19, 20]. For rotor balancing problem, deep learning technologies are usually used to address the input identification problem by learning the inverse mapping from data, and the learning of the mapping can be processed by same data used for balancing process. That means, no extra data or prior knowledge are needed for rotor balancing. Based on this truth, in this work, we are going to propose a novel balancing method for rotor with deep learning network. In the following, we provide an overview about the related work in Section 2. In Section 3, the proposed method is illustrated briefly. To prove the validity of the proposed method, a case study is undertaken in Section 4. Finally, the paper is concluded.

\section{Related Work}

Rotor balancing methods have been developed from various perspectives including algorithm modification and introduction of advanced technology, for example, Bin et al. $[21,22]$ investigated and balanced a kind of multirotor turbomachinery shaft system with $N+1$ supports analytically and experimentally. Zhao et al. [23] proposed a transient characteristic-based balancing method (TCBM) combined with dynamic load identification (DLI) technique to identify the unbalance parameters of the general rotor system. Li et al. [24] proposed a novel modal balancing technique without trial weights by combining the modal balancing method with finite elements method. Zhang et al. $[25,26]$ identified an unbalance response of a dual-rotor system with a slight rotating speed difference by the wholebeat correlation method and nonwhole-beat correlation method, respectively. Yue et al. [27] presented an innovative modal balancing process for estimating the residual unbalance from different equilibrium planes of complex flexible rotor system. Yu et al. [28] proposed a new adaptive proportional-integral control strategy for rotor active balancing systems during acceleration. Li et al. [29] proposed a novel disturbance-observer-based field dynamic balancing strategy for active magnetic bearings (AMB) equipped machinery. The imbalance distribution of the rotor may lead to vibrations and bifurcations in rotor-active magnetic bearing systems [30, 31]. Zheng and Wang [32] presented a novel high-precision field balancing method based on the regular control mode without trial weight. Ait Ben Ahmed et al. [33] presented and validated a hybrid method through a series of experiments for balancing of rigid and flexible rotors at a constant rotational speed.

Learning the model from data has been addressed in the references by either system identification or supervised black-box function approximation. Ting et al. [34] developed a Bayesian parameter identification method that can automatically detect noise in both input and output data for the regression algorithm that performs system identification. Atkeson et al. [35] presented a procedure that estimates load and link inertial parameters as a result of general movement; the loads and links are considered to be rigid bodies. Haruno et al. [36] proposed a new modular architecture, the modular selection and identification for control (MOSAIC) model, for motor learning and control based on multiple pairs of forward (predictor) and inverse (controller) models. Calinon et al. [37] presented and evaluated an approach based on the hidden Markov model (HMM), Gaussian mixture regression (GMR), and dynamical systems to allow robots to acquire new skills by imitation. Ledezma and Haddadin [38] introduced a conceptual framework for the construction and training of first-order principle (FOP) networks and described the proposed estimation method in detail. In contrast to these existing methods, the proposed method is going to learn the mapping from system state to bearing force directly, while the unbalanced forces are to be learned unsupervised.

\section{Proposal of the Method}

3.1. Problem Formulation. Describing the equations of motion for mechanical systems has been extensively studied, and various formalism to derive these equations exists. The most prominent are Newtonian-, Hamiltonian-, and Lagrangian-mechanics [39]. Within this work, Newtonianmechanics is used, and based on which, a mapping model $f$ can be used either to predict the change of system state in forward way or identify the input $\tau$ in its inverse form $f^{-1}$, i.e.,

$$
\begin{aligned}
f(\mathbf{q}, \dot{\mathbf{q}}, \boldsymbol{\tau}) & =\ddot{q}, \\
f^{-1}(\mathbf{q}, \dot{\mathbf{q}}, \ddot{\mathbf{q}}) & =\boldsymbol{\tau},
\end{aligned}
$$

where $\mathbf{q}$ refers to the generalized coordinate vector, and $\dot{\mathbf{q}}$ and $\ddot{\mathbf{q}}$ are corresponding generalized velocity and acceleration vectors. Approximating $f$ and $f^{-1}$ with deep networks, i.e.,

$$
\begin{aligned}
\widehat{f} & =\widehat{f}(\mathbf{q}, \dot{\mathbf{q}}, \boldsymbol{\tau} ; \boldsymbol{\alpha}), \\
\widehat{f}^{-1} & =\widehat{f}^{-1}(\mathbf{q}, \dot{\mathbf{q}}, \ddot{\mathbf{q}} ; \boldsymbol{\beta}),
\end{aligned}
$$

where $\hat{\cdot}$ means an approximation and $\alpha$ and $\beta$ are network parameter tensors' the forward model and inverse model could be learned from data. For an identification problem, the inverse model learns the mapping from joint configuration $(\mathbf{q}, \dot{\mathbf{q}}, \ddot{\mathbf{q}})$ to input force $\tau$. In detail, the network parameters can be learned by minimizing the error between the real value of sample and the predict value, using the samples $(\mathbf{q}, \dot{\mathbf{q}}, \ddot{\mathbf{q}}, \tau)$ obtained from the mechanical system. For the rotor balancing problem, the input force can be obtained from state variables and their derivatives in different time intervals or at different time points. So, abundant training data can be obtained by just one run of the rotor system. Weight trail processes are not essential in the proposed method. 
3.2. Unsupervised Deep Network for Unbalanced Force. Starting from (1), supervised learning approaches would train the parameters by minimizing the $\ell_{i}$ norm between the prediction of (1) and measured input force $\tau$, i.e., by solving a optimization problem described as follows:

$$
\boldsymbol{\beta}^{*}=\underset{\boldsymbol{\beta}}{\operatorname{argmin}} \ell_{i}\left(\hat{f}^{-1}(\mathbf{q}, \dot{\mathbf{q}}, \ddot{\mathbf{q}} ; \beta), \boldsymbol{\tau}\right) .
$$

In rotor balancing problem, as the unbalanced force cannot be measured directly, if one would like to learn the unbalanced force supervised, many runs with preset unbalanced mass have to be done. This obeys our aim. Thus, in this section, an unsupervised deep network to predict unbalanced forces is established. The proposed network is shown in Figure 1.

In the rotor system, vibration displacements of the disk and shaft can be easily measured by eddy current sensors, while the velocity and acceleration cannot. Thus, a preprocessing module is introduced to derive the measured displacement. Meanwhile, the unbalanced force cannot be obtained. But the bearing force can be obtained indirectly through the bearing support as

$$
\boldsymbol{\tau}_{\mathrm{sm}}=\mathbf{m a}-\mathbf{F},
$$

where $\mathbf{m}$ is the mass matrix of the bearing support, $\mathbf{a}$ is the acceleration vector of the bearing support, and $\mathbf{F}$ is the force vectors measured by dynamic force sensors.

In this situation, the optimization problem can be described by slight modification of (3) to

$$
\boldsymbol{\beta}^{*}=\underset{\boldsymbol{\beta}}{\operatorname{argmin}} L\left(\left(\widehat{f}^{-1}(\mathbf{q}, \dot{\mathbf{q}}, \ddot{\mathbf{q}} ; \beta)\right)_{s}, \boldsymbol{\tau}_{\mathrm{sm}}\right),
$$

where $L$ can be arbitrary loss function.

In Figure 1, the neutral network module can be arbitrary type, changing the 2-dimensional input data into 1-dimensional output data. The dimension of output data should be the same with the state-dimensional dimension of the input data. This module is going to convert the shape of the input data and increase the complexity of the proposed network. After the neutral network module, three 1-dimensional convolutional layers are applied. The first one has 128 filters with a size of 1 by 3 . The convolutional calculation is applied to the output from the network module. After the feature extraction process, the low order representations of the data are obtained. The Relu activation function is applied, so that the low order representations are activated. The second convolutional layer has 64 filters with a size of 1 by 3 . Then, the high order representations are extracted and activated. The third convolutional layer has just one filter with the size determined by the dimension of the expected input force, i.e., the concatenation of dimension of the indirectly measured bearing force vector $\tau_{\mathrm{sm}}$ and dimension of the unknown unbalanced force vector $\tau_{s}$. Thus, the final output can be expected as input data, in which $\tau_{s}$ is used to solve the optimization problem described by (5) and $\tau_{u}$ is used as the prediction of the unbalanced force. It is noting that the parameter sharing mechanism of convolutional networks ensure that all the trainable variables in proposed network would be updated during the back propagation process. Therefore, the proposed network learns the inverse mapping from labeled $\tau_{\mathrm{sm}}$ and learns $\tau_{u}$ unsupervisedly.

3.3. Procedure Description. The overview of the proposed method is shown in Figure 2. As Figure 2 illustrated, there are three steps for the whole balancing method. Firstly, the data obtained from the system should be preprocessed, i.e., the displacements should be derived to get the velocities and accelerations and bearing forces are obtained from the measured support forces by equation (4). Second, the proposed network is set up. The architecture and the untrainable parameters should be determined. Based on the loss function chosen, the training process is done by back propagation. The trainable variables are updated until the standard of the optimization is met. Third, the predicted unbalanced force should be fit to get the amplitude and phase of the harmonic type of force. Based on the identification results, the weight adding and weight reducing processes should be conducted on the rotor. An additional test to verify the performance of the balancing is necessary obviously.

\section{Case Study}

4.1. Numerical Model Description. In this section, a general double disc rotor with preset imbalances is used to test the method as a case study. Figure 3 illustrates the dynamical model of the rotor consisting of two mass discs and two sliding bearings.

Considering the gravity, unbalanced force, gyroscopic effect, and bearing force, the dynamic model of the rotor support structure shown in Figure 3 can be established into form:

$$
\mathbf{M} \ddot{\mathbf{q}}+(\mathbf{C}+\Omega \mathbf{G}) \dot{\mathbf{q}}+\mathbf{K q}+\mathbf{F}_{n}=\mathbf{F}_{u}+\mathbf{F}_{g},
$$

where $\mathbf{q}$ is the displacement coordinate vector, including the displacements of the two disks and two sliding bearings along the $x$-axis and $y$-axis, respectively; $\mathbf{M}, \mathbf{C}, \mathbf{G}$, and $\mathbf{K}$ are the corresponding mass matrix, damping matrix, gyro matrix, and stiffness matrix; $\mathbf{F}_{n}$ is the bearing force vector; $\mathbf{F}_{u}$ is the unbalanced force vector; and $\mathbf{F}_{g}$ is the gravity vector. $\Omega$ represents the rotating speed of the rotor which determines the frequency of the unbalanced force.

Reynolds' equation is the basic equation for oil film force analysis:

$$
\frac{1}{R^{2}} \cdot \frac{\partial}{\partial \zeta}\left(\frac{h^{3}}{12 \eta} \cdot \frac{\partial p}{\partial \zeta}\right)+\frac{\partial}{\partial z}\left(\frac{h^{3}}{12 \eta} \cdot \frac{\partial p}{\partial z}\right)=\frac{1}{2} \Omega \cdot\left(\frac{\partial h}{\partial \zeta}+\frac{\partial h}{\partial t}\right),
$$

where $R$ is the radius of the journal; $\zeta$ is the clockwise angle from vertical direction; $h$ is the oil film thickness; $\eta$ is the lubricating oil viscosity; and $p$ is the oil film pressure. $z$ indicates axial-direction which is neglected in this study and $t$ for time. 


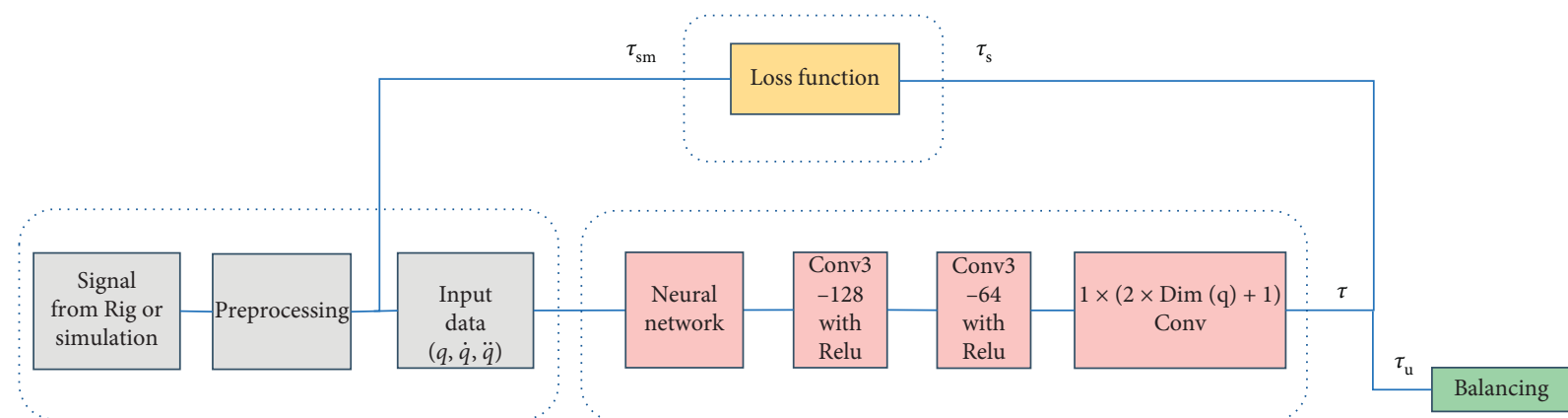

FIgURE 1: Overview of the unsupervised network, where Conv3-128 means a convolutional layer with 128 filters of size $1 \times 3$ and Relu means rectified linear unit activation function.

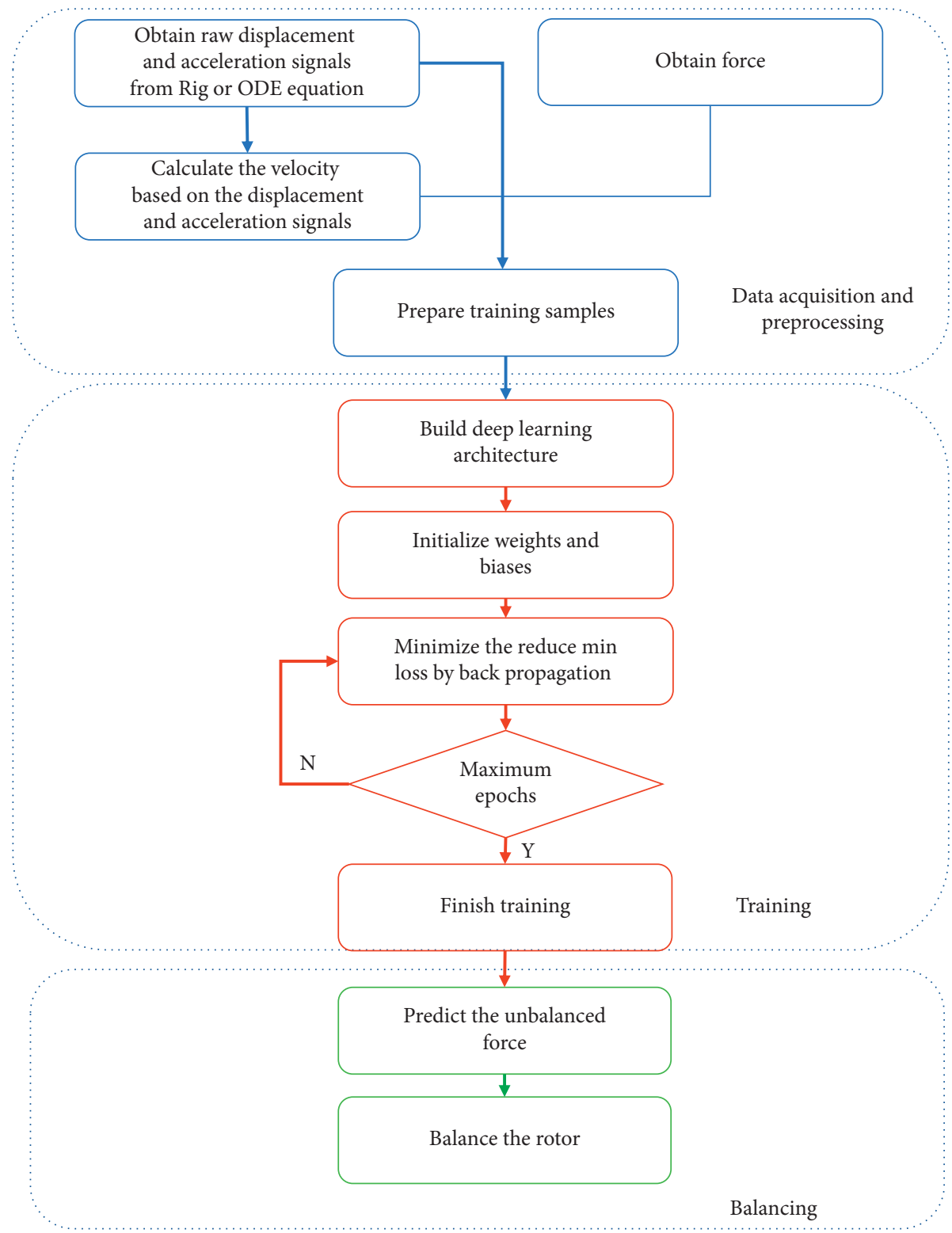

Figure 2: The flow chart of the proposed method. 


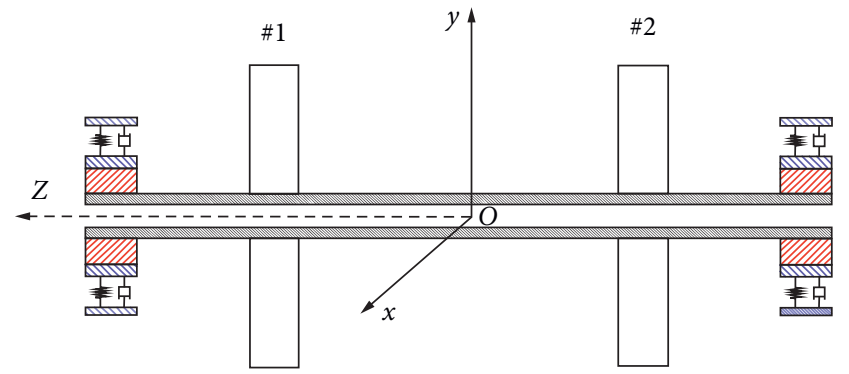

Figure 3: Schematic diagram.

Capone's modified oil film force model [40] is adopted based on which (7) can be solved analytically. Then, the oil film forces are obtained with the help of Capone's simplification.

$$
\begin{aligned}
\left\{\begin{array}{c}
f_{x} \\
f_{y}
\end{array}\right\}= & \left.\frac{\left[\left(x-2 y^{\prime}\right)^{2}+\left(y+2 x^{\prime}\right)^{2}\right]^{1 / 2}}{1-x^{2}-y^{2}}\right) \\
& \cdot\left\{\begin{array}{l}
3 x V(x, y, \alpha)-\sin \alpha G(x, y, \alpha)-2 \cos \alpha S(x, y, \alpha) \\
3 y V(x, y, \alpha)+\cos \alpha G(x, y, \alpha)-2 \sin \alpha S(x, y, \alpha)
\end{array}\right\},
\end{aligned}
$$

where

$$
\begin{aligned}
V(x, y, \alpha)= & \frac{2+(y \cos \alpha-x \sin \alpha) G(x, y, \alpha)}{1-x^{2}-y^{2}} \\
S(x, y, \alpha)= & \frac{x \cos \alpha+y \sin \alpha}{1-(x \cos \alpha+y \sin \alpha)^{2}}, \\
G(x, y, \alpha)= & \frac{2}{\left(1-x^{2}-y^{2}\right)^{1 / 2}}\left[\frac{\pi}{2}+\arctan \frac{y \cos \alpha-x \sin \alpha}{\left(1-x^{2}-y^{2}\right)^{1 / 2}}\right] \\
\alpha= & \arctan \frac{y+2 x^{\prime}}{x-2 y^{\prime}}-\frac{\pi}{2} \operatorname{sgn}\left(\frac{y+2 x^{\prime}}{x-2 y^{\prime}}\right) \\
& -\frac{\pi}{2} \operatorname{sgn}\left(y+2 x^{\prime}\right)
\end{aligned}
$$

where subscript $x$ indicates the horizontal component of the oil film force in the radial direction and $y$ indicates the vertical component of the oil film force in the radial direction. $x, y, x^{\prime}$, and $y^{\prime}$ are the general displacements and velocities of corresponding directions.

4.2. Identification of the Unbalanced Force. As a case study, training dataset is prepared by doing the dynamical simulation of the numerical model described by equation (6). The initial values of the state variables are given as zeros. With the expressions of bearing forces described by equation (8) and preset unbalanced masses, the state variables can be obtained by numerical integration method with time evolution and the general accelerations and corresponding bearing forces can be obtained by solving the equations (6) and (8) algebraically.

The data are prepared in a scaled time span from 0 to 100 , with an interval of 0.01 , i.e., $(0,100,0.01)$. Therefore, the length of the dataset is fixed to 10000 . In this study, each sample's length is set to 1 . That means, the number of training data will be 10000 . The joint configurations $(\mathbf{q}, \dot{\mathbf{q}}, \ddot{\mathbf{q}})$ took out from the training dataset will be input to the proposed network. And, the $\tau_{s}$-dimensional outputs are compared with the $\tau_{\text {sm }}$ by loss function for parameters update. Mean squared error (MSE) of $\tau_{\text {sm }}$ and $\tau_{s}$ is used as optimization objection. Minibatch of 128 and Adam optimizer are applied during the training process.

After 1000 epochs, the learning results of the bearing forces under supervision are given in Figure 4. The red lines in Figures 4(a)-4(d) represent the values of bearing force of right end and left end bearings in horizontal and vertical directions respectively, which are calculated by equation (8). The black dotted lines are corresponding outputs of the proposed network.

The prediction values of the unbalanced forces are shown as blue asterisks in Figure 5. As the unbalanced force is assumed to be of harmonic type, the first guess (green lines in Figure 5) follow the rule of

$$
\text { g_std } * \sin \left(t+g_{-} \text {pha }\right)+g_{-} \text {mean, }
$$

where $g_{-}$mean $=$Mean $($data $), g_{-}$std $=3 \sqrt{2} / 2 \operatorname{Std}($ data $)$, and $g$-pha $=0$. The least square method is, then, used to fit the amplitude and the phase of the unbalanced force. The identification results (orange lines in Figure 5) can be used to balance the rotor.

Compared with the ICM, the identification results are listed in Table 1 . The preset values are randomly chosen. Because of the nonlinearties introduced by the bearing force, the identification results by ICM are not closed to the preset values, while the proposed values are close to the preset value.

4.3. Balancing Results. Based on the identification results of the amplitudes and the phases of the unbalanced forces, the rotor system with preset imbalances established in case study can be balanced. The effects are illustrated in Figure 6. In Figure 6, the red lines represent the responses of the left bearing, the left disk, the right disk, and the right bearing. The amplitudes of the responses are huge. At some points, the value is bigger than 1 , which indicates rub-impact faults should happen. Also, the shape of the responses before balancing indicates that, besides the base frequency vibrations, there are other frequency components which exist. After balancing, the response curves (black broken lines) become smooth, and the amplitudes reduce significantly. 

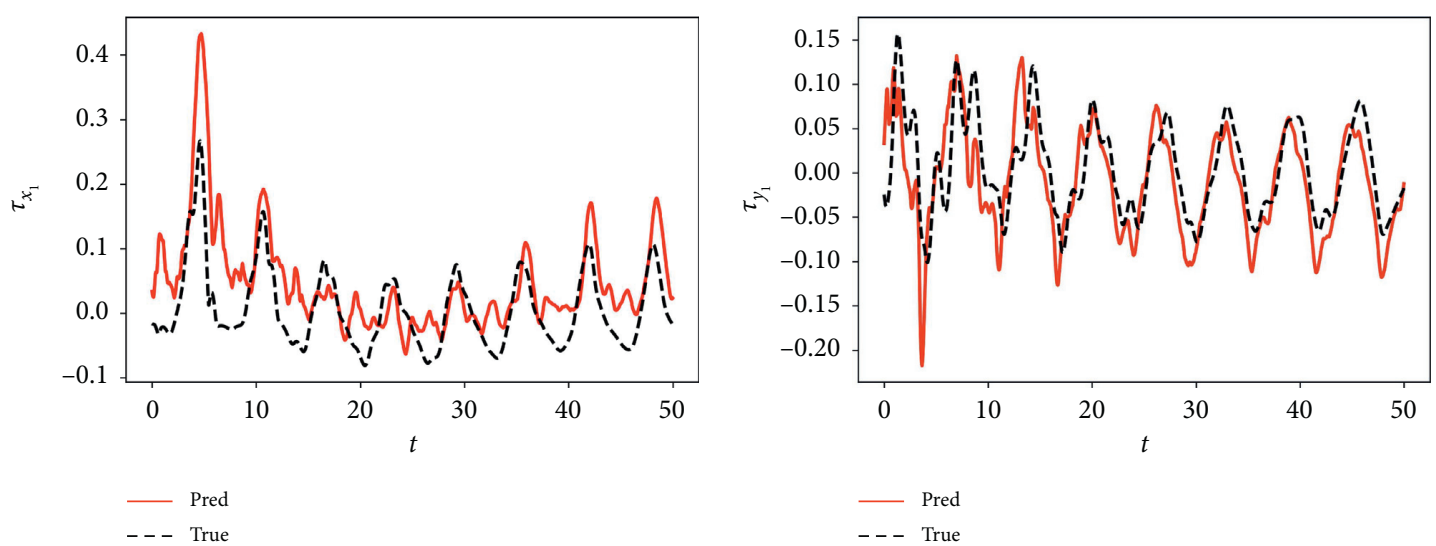

(a)

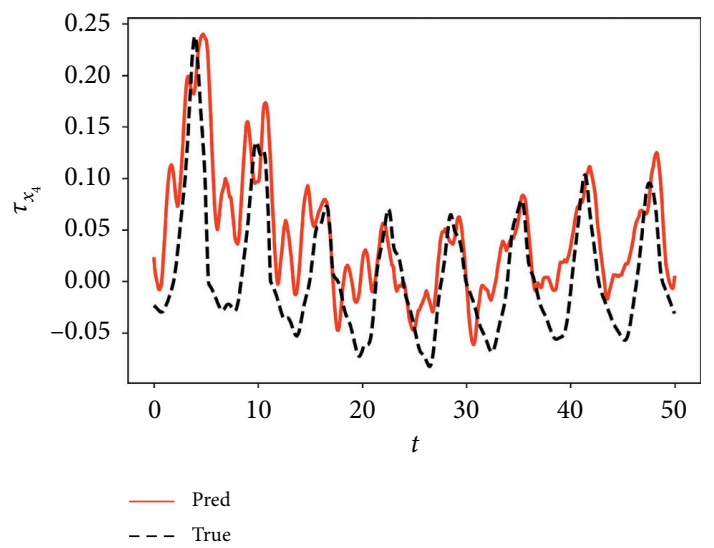

(c)

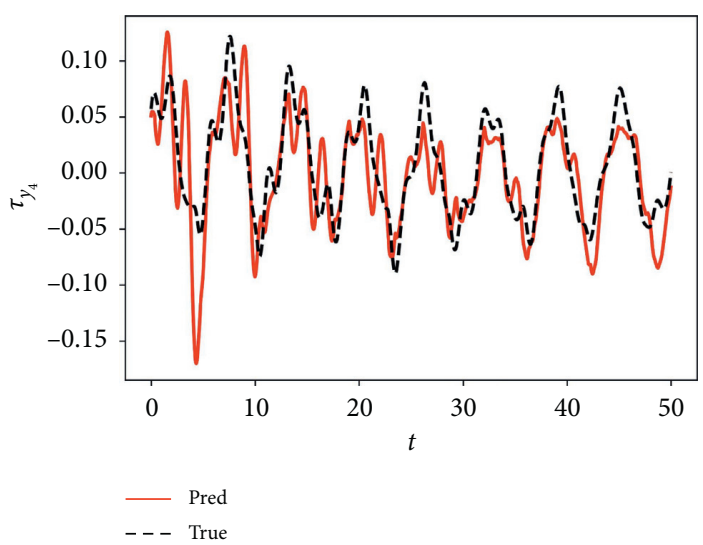

(d)

FIgURE 4: The prediction values of the bearing forces after the training process: (a) horizontal component of bearing force at the left end; (b) vertical component of the bearing force at the left end; (c) horizontal component of the bearing force at the right end; and (d) vertical component of the bearing force at the right end.

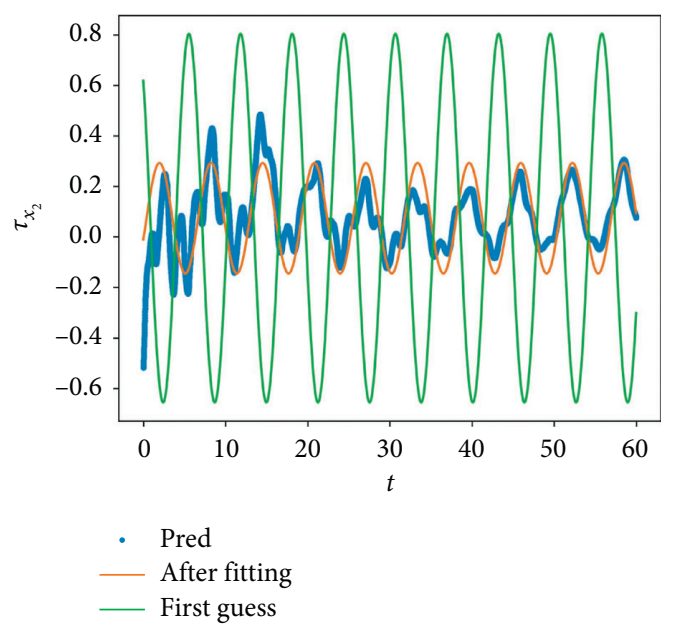

(a)

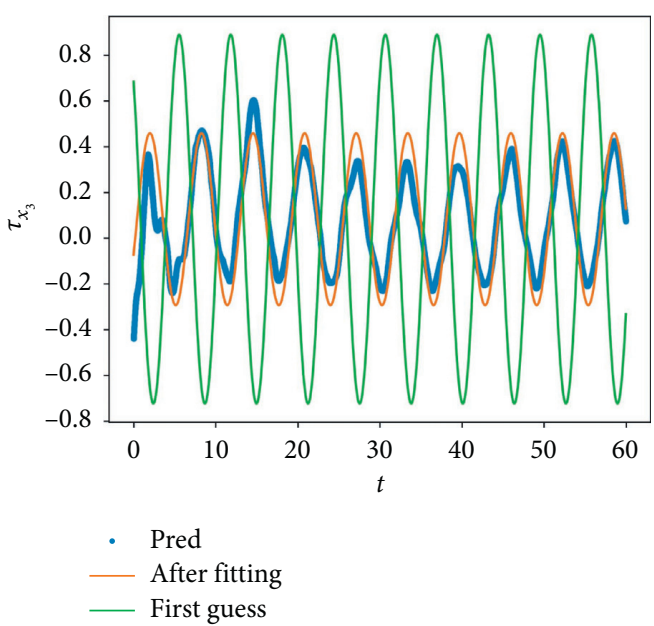

(b)

FIgURE 5: The prediction results of the unbalanced forces and their harmonic fits: (a) the results at correction plane 1 and (b) correction plane 2 . 
TABLE 1: The identification results of the unbalanced forces.

\begin{tabular}{lccc}
\hline & Preset value & ICM & Proposed method \\
\hline Amplitude of imbalance at plane 1 & 0.294 & 0.0891 & 0.2219 \\
Phase of imbalance at plane 1 & $\pi / 2$ & 0.2827 & 2.1432 \\
Amplitude of imbalance at plane 2 & 0.3125 & 0.6259 & 0.376 \\
Phase of imbalance at plane 1 & $\pi / 3$ & 0.6746 & 1.339 \\
\hline
\end{tabular}

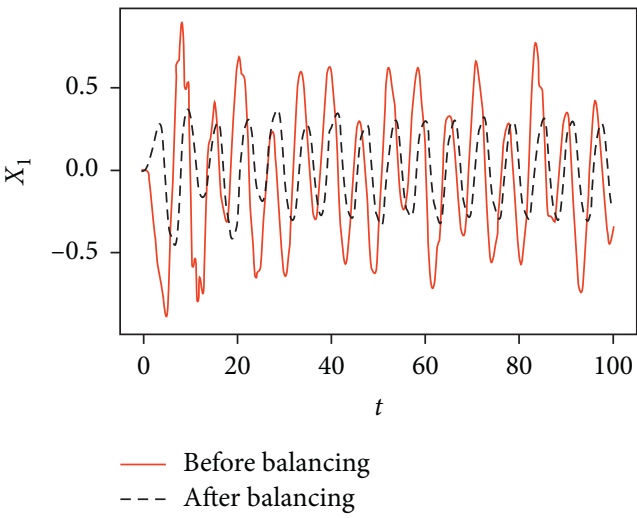

(a)

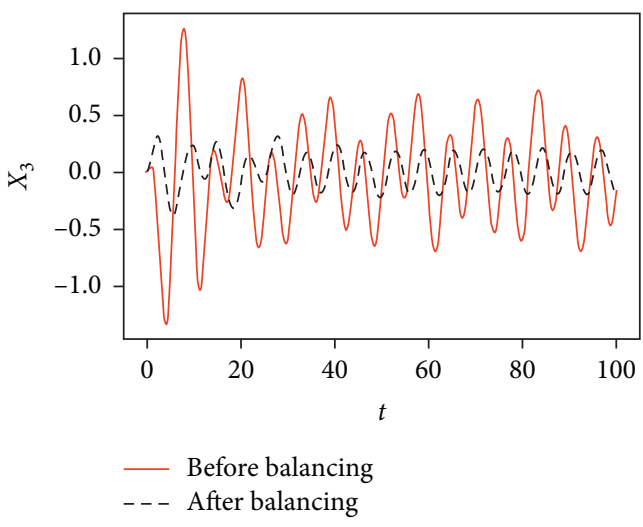

(c)

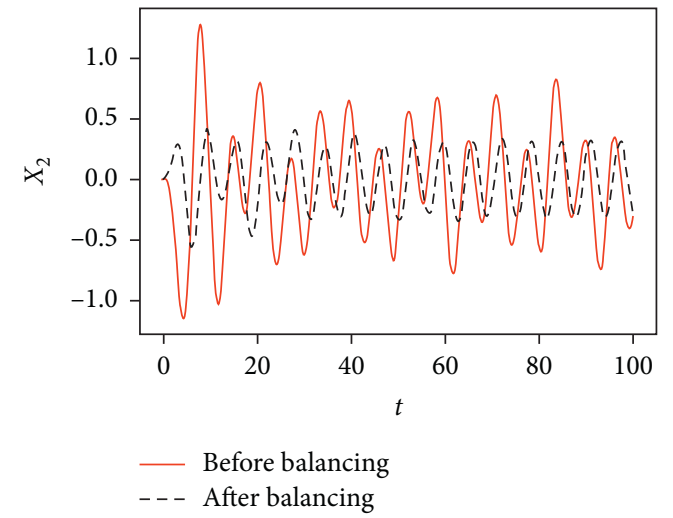

(b)

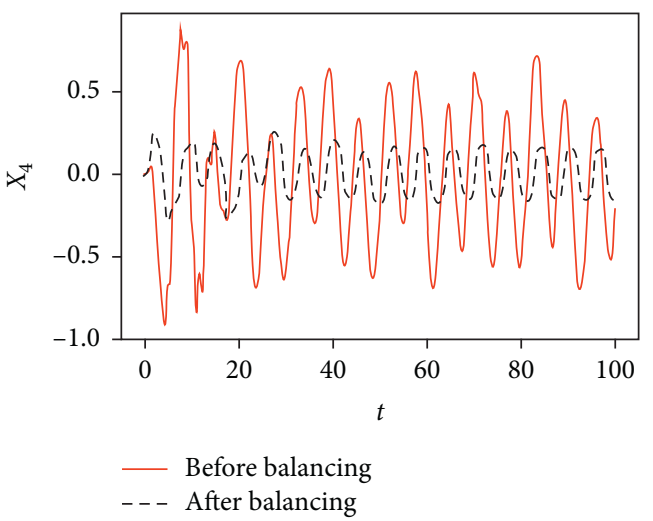

(d)

Figure 6: Balancing effects on the (a) left end; (b) left disk; (c) right disk; and (d) right end.

\section{Discussion and Conclusions}

A novel rotor balancing method using unsupervised deep learning is proposed in this work. The method uses joint configurations of the general displacements, velocities, and accelerations to learn the unbalanced forces, thereby realizing a balancing process without weight trails. Compared to the supervised control, the proposed method used the parameter sharing mechanism of convolutional networks to learn the unbalanced forces unsupervisedly. A mechanical model of a two-disc rotor with preset imbalances is balanced as a case study. The balancing results prove that the proposed method considers both cost and accuracy at the same time. The dynamic behaviours of the rotor after balancing are reasonable.

It is nothing that, in the proposed network and training process, there are many parameters which can be optimized to achieve better performances. For instance, physical prior layer can be added to the neutral network module to introduce physical meaning of the mechanical system. The size of a single sample can be enlarged during data preprocessing, so that more historic information could be involved. All these aspects deserve further study.

\section{Data Availability}

No public dataset has been used in the manuscript, and all the data used to draw the figures and support the conclusions of the manuscript are generated by calculating the model established in the manuscript. The calculation result data used to support the findings of this study are available from the first author (Shun Zhong) upon request. All data will be released, including codes, after acceptance of the manuscript.

\section{Conflicts of Interest}

The authors declare no conflicts of interest. 


\section{Acknowledgments}

This work was supported by the National Natural Science Foundation of China (grant nos. 11502161 and 11902184).

\section{References}

[1] J. Yao, F. Yang, Y. Su, F. Scarpa, and J. Gao, "Balancing optimization of a multiple speeds flexible rotor," Journal of Sound and Vibration, vol. 480, Article ID 115405, 2020.

[2] Y. Cao, F. Li, J. Cao, and T. Wang, "Calibration of a hub dynamic balancing machine based on the least squares method and systematic error analysis," IEEE Access, vol. 8, pp. 178746-178754, 2020.

[3] G. Ranjan and R. Tiwari, "On-site high-speed balancing of flexible rotor-bearing system using virtual trial unbalances at slow run," International Journal of Mechanical Sciences, vol. 183, Article ID 105786, 2020.

[4] S. Zhang and Z. Zhang, "Online measuring and estimating methods for the unbalancing vector of thin-disc workpiece based on the adaptive influence coefficient," Journal of Vibration and Control, vol. 27, pp. 1-12, 2020.

[5] R. E. D. Bishop and G. M. L. Gladwell, "The vibration and balancing of an unbalanced flexible rotor," Journal of Mechanical Engineering Science, vol. 1, no. 1, pp. 66-77, 1959.

[6] R. E. D. Bishop and A. G. Parkinson, "On the use of balancing machines for flexible rotors," Journal of Manufacturing Science and Engineering, vol. 94, no. 2, pp. 561-572, 1972.

[7] T. P. Goodman, "A least-squares method for computing balance corrections," Journal of Manufacturing Science and Engineering, vol. 86, no. 3, pp. 273-277, 1964.

[8] J. W. Lund and J. Tonnesen, "Analysis and experiments on multi-plane balancing of a flexible rotor," Journal of Manufacturing Science and Engineering, vol. 94, no. 1, pp. 233-242, 1972.

[9] C. D Untaroiu, P. E. Allaire, and W. C. Foiles, "Balancing of flexible rotors using convex optimization techniques: optimum min-max lmi influence coefficient balancing," Journal of Vibration and Acoustics, vol. 130, no. 2, Article ID 021006, 2008.

[10] X. Wang, "SQP algorithms in balancing rotating machinery," Mechanical Systems and Signal Processing, vol. 21, no. 3, pp. 1469-1478, 2007.

[11] T. Messager and M. Pyrz, "Discrete optimization of rigid rotor balancing," Journal of Mechanical Science and Technology, vol. 27, no. 8, pp. 2231-2236, 2013.

[12] Y. Kang, T.-W. Lin, Y.-J. Chang, Y.-P. Chang, and C.-C. Wang, "Optimal balancing of flexible rotors by minimizing the condition number of influence coefficients," Mechanism and Machine Theory, vol. 43, no. 7, pp. 891-908, 2008.

[13] G. Li, Z. Lin, and P. E. Allaire, "Robust optimal balancing of high-speed machinery using convex optimization," Journal of Vibration and Acoustics, vol. 130, no. 3, Article ID 031008, 2008.

[14] S. Tresser, A. Dolev, and I. Bucher, "Dynamic balancing of super-critical rotating structures using slow-speed data via parametric excitation," Journal of Sound and Vibration, vol. 415, pp. 59-77, 2018.

[15] Y. A. Khulief, W. Oke, and M. A. Mohiuddin, "Modally tuned influence coefficients for low-speed balancing of flexible rotors," Journal of Vibration and Acoustics, vol. 136, no. 2, Article ID 024501, 2014.
[16] M. B. Deepthikumar, A. S. Sekhar, and M. R. Srikanthan, "Modal balancing of flexible rotors with bow and distributed unbalance," Journal of Sound and Vibration, vol. 332, no. 24, pp. 6216-6233, 2013.

[17] M. Villafane Saldarriaga, V. Steffen, J. Der Hagopian, and J. Mahfoud, "On the balancing of flexible rotating machines by using an inverse problem approach," Journal of Vibration and Control, vol. 17, no. 7, pp. 1021-1033, 2011.

[18] D.-J. Han, "Generalized modal balancing for non-isotropic rotor systems," Mechanical Systems and Signal Processing, vol. 21, no. 5, pp. 2137-2160, 2007.

[19] Y. Zhang, J. Ji, and B. Ma, "Fault diagnosis of reciprocating compressor using a novel ensemble empirical mode decomposition-convolutional deep belief network," Measurement, vol. 156, Article ID 107619, 2020.

[20] G. F. Bin, J. J. Gao, X. J. Li, and B. S. Dhillon, "Early fault diagnosis of rotating machinery based on wavelet packetsEmpirical mode decomposition feature extraction and neural network," Mechanical Systems and Signal Processing, vol. 27, pp. 696-711, 2012.

[21] G. Bin, X. Li, Y. Shen, and W. Wang, "Development of wholemachine high speed balance approach for turbomachinery shaft system with $\mathrm{N}+1$ supports," Measurement, vol. 122, pp. 368-379, 2018.

[22] G. Bin, X. Li, J. Wu, and J. Gao, "Virtual dynamic balancing method without trial weights for multi-rotor series shafting based on finite element model analysis," Journal of Renewable and Sustainable Energy, vol. 6, no. 4, Article ID 042014, 2014.

[23] S. Zhao, X. Ren, W. Deng, K. Lu, Y. Yang, and C. Fu, "A transient characteristic-based balancing method of rotor system without trail weights," Mechanical Systems and Signal Processing, vol. 148, Article ID 107117, 2021.

[24] X. Li, L. Zheng, and Z. Liu, "Balancing of flexible rotors without trial weights based on finite element modal analysis," Journal of Vibration and Control, vol. 19, no. 3, pp. 461-470, 2013.

[25] Z. X. Zhang, Q. Zhang, X. L. Li, and T. L. Qian, “The wholebeat correlation method for the identification of an unbalance response of a dual-rotor system with a slight rotating speed difference," Mechanical Systems and Signal Processing, vol. 25, no. 5, pp. 1667-1673, 2011.

[26] Z. X. Zhang, L. Z. Wang, Z. J. Jin, Q. Zhang, and X. L. Li, "Non-whole beat correlation method for the identification of an unbalance response of a dual-rotor system with a slight rotating speed difference," Mechanical Systems and Signal Processing, vol. 39, no. 1-2, pp. 452-460, 2013.

[27] C. Yue, X. Ren, Y. Yang, and W. Dang, "Unbalance Identification of speed-variant rotary machinery without phase angle measurement," Shock and Vibration, vol. 2015, Article ID 934231, 11 pages, 2015.

[28] X. Yu, K. Mao, S. Lei, and Y. Zhu, "A new adaptive proportional-integral control strategy for rotor active balancing systems during acceleration," Mechanism and Machine Theory, vol. 136, pp. 105-121, 2019.

[29] K. Li, C. Peng, Z. Deng, W. Huang, and Z. Zhang, "Field dynamic balancing for active magnetic bearings supporting rigid rotor shaft based on extended state observer," $\mathrm{Me}$ chanical Systems and Signal Processing, vol. 158, Article ID 107801, 2021.

[30] J. C. Ji, C. H. Hansen, and A. C. Zander, "Nonlinear dynamics of magnetic bearing systems," Journal of Intelligent Material Systems and Structures, vol. 19, no. 12, pp. 1471-1491, 2008. 
[31] J. C. Ji and C. H. Hansen, "Non-linear oscillations of a rotor in active magnetic bearings," Journal of Sound and Vibration, vol. 240, no. 4, pp. 599-612, 2001.

[32] S. Zheng and C. Wang, "Rotor balancing for magnetically levitated tmps integrated with vibration self-sensing of magnetic bearings," IEEE/ASME Transactions on Mechatronics, 2021.

[33] A. Ait Ben Ahmed, A. Touache, A. El Hakimi, and A. Chamat, "A new hybrid method for rigid and flexible rotor balancing without phase response measurements," Australian Journal of Mechanical Engineering, pp. 1-13, 2020.

[34] J. A. Ting, M. N. Mistry, J. Peters, S. Schaal, and J. Nakanishi, "A bayesian approach to nonlinear parameter identification for rigid body dynamics," in Proceedings of the Robotics: Science and Systems, pp. 32-39, Philadelphia, PA, USA, August 2006.

[35] C. G. Atkeson, C. H. An, and J. M. Hollerbach, "Estimation of inertial parameters of manipulator loads and links," The International Journal of Robotics Research, vol. 5, no. 3, pp. 101-119, 1986.

[36] M. Haruno, D. M. Wolpert, and M. Kawato, "Mosaic model for sensorimotor learning and control," Neural Computation, vol. 13, no. 10, pp. 2201-2220, 2001

[37] S. Calinon, F. D’halluin, E. Sauser, D. Caldwell, and A. Billard, "Learning and reproduction of gestures by imitation," IEEE Robotics and Automation Magazine, vol. 17, no. 2, pp. 44-54, 2010.

[38] F. Ledezma and S. Haddadin, "First-order-principles-based constructive network topologies: an application to robot inverse dynamics," in Proceeding of the IEEE-RAS 17th International Conference on Humanoid Robotics (Humanoids), pp. 438-445, IEEE, Brimingham, England, November 2017.

[39] M. Lutter, K. Listmann, and J. Peters, "Deep lagrangian networks for end-to-end learning of energy-based control for under-actuated systems," 2019, https://arxiv.org/abs/1907. 04489.

[40] G. Capone, "Analytical description of fluid-dynamic force field in cylindrical journal bearing," LâẤźEnergia Elettrica, vol. 3, no. 3, pp. 105-110, 1991. 